\title{
EMDR Treatment of Comorbid PTSD and Alcohol Dependence: A Case Example
}

\author{
Nancy J. Abel \\ South Portland, Maine \\ John M. O’Brien \\ Portland, Maine
}

\begin{abstract}
Eye movement desensitization and reprocessing (EMDR) is a therapy that has been demonstrated to be effective in the treatment of posttraumatic stress disorder (PTSD). A relatively small but growing body of literature indicates that EMDR may be an effective adjunctive treatment for substance abuse. This article reviews the various protocols that have been developed for that purpose, including protocols by Vogelmann-Sine et al., Omaha, Popky, and Hase. A case study that incorporates the use of some of these interventions is presented to illustrate successful EMDR treatment of a woman who had longstanding comorbid alcohol abuse and PTSD. Two-year follow-up after EMDR showed that the woman was successfully maintaining sobriety and that the PTSD continued in full remission. After a discussion of the important aspects of this case, the authors explore future directions for research.
\end{abstract}

Keywords: EMDR; substance abuse; alcohol dependency; treatment; PTSD; comorbid disorders

E ye movement desensitization and reprocessing (EMDR) is a therapy first developed in 1987 by Francine Shapiro for the treatment of traumatic memories (Shapiro, 1989). Since its inception, EMDR has grown from a desensitization technique into an integrated psychotherapeutic treatment approach (Solomon \& Shapiro, 2008). EMDR has now established its efficacy for the treatment of both acute stress disorder and posttraumatic stress disorder (PTSD; American Psychiatric Association [APA], 2004). The most recent version of practice guidelines for the International Society for Traumatic Stress Studies cites cognitive-behavioral treatments (including exposure therapy and cognitive-processing therapy) and EMDR as among the PTSD treatments evidencing the greatest empirical support (Foa, Keane, Friedman, \& Cohen, 2009). Recent research comparing EMDR to traditional exposure-based treatments of trauma has often found EMDR to be equally or more efficacious (Ironson, Freund, Strauss, \& Williams, 2002; Lee, Taylor, \& Drummond, 2006).

Although the extant literature describes EMDR treatment for numerous other presenting complaints, including various anxiety disorders, couples' conflicts, and issues with performance (Shapiro \& Forrest, 1997), research of its effectiveness in these approaches is still lacking. There is, however, some preliminary evidence that EMDR may be effective as an adjunctive therapy in the treatment of substance use disorders. EMDR's possible utility with these disorders may stem from the high levels of traumatic exposure and PTSD in this population. The incidence of traumatic exposure in individuals with substance use disorders has been well established in the field (Peirce, Kindbom, Waesche, Yuscavage, $\&$ Brooner, 2008). Research has indicated that $22 \%-43 \%$ of people living with PTSD have a lifetime prevalence rate of substance use disorders and that the rate for veterans is as high as $75 \%$ (Jacobsen, Southwick, \& Kosten, 2001). Literature indicates the complex nature of treating patients with comorbid PTSD and substance abuse. These clients often have greater difficulty maintaining sobriety as well as increased difficulty in the healing of traumatic memories (Ford, Hawke, Alessi, Ledgerwood, \& Petry, 2007; Peirce, Kindbom, Waesche, Yuscavage, \& Brooner, 2008; Schumacher, Coffey, \& Stasiewicz, 2006).

Many approaches have been tried in the treatment of addiction (van Wormer \& Davis, 2008). Clients 
may utilize some combination of medications, detoxification services, individual therapy, family therapy, group therapy, and/or self-help groups, such as Alcoholics Anonymous (AA), depending on individual needs (Allen $\&$ Litten, 1999). Despite these options and attempts to develop new ways to deal with addiction, relapse rates can be as high as 90\% (Gracer, 2007; Marlatt \& Gordon, 1985). Clinicians and researchers continue to develop treatment protocols that will be most effective in working with diverse clients. Project Match (1997) paired specific clients with specific treatment approaches. It was found that all three treatments reviewed worked effectively with clients, regardless of background. More recent treatments have incorporated a "harm reduction" approach, acknowledging that both abstinence and reduction in substance use can be viewed as a successful treatment outcome (Marlatt \& Gordon, 1998).

\section{Treatment of Addiction With EMDR}

Shapiro, Vogelmann-Sine, and Sine (1994) reported on the early uses of EMDR with addictions at all stages of substance abuse treatment. These authors recommended that "for optimal therapeutic effects, the client should be personally and systematically stabilized, engaged in a 12-step support group, and abstinent for a sufficient period of time to prevent physical symptoms of withdrawal" (p. 379). They suggested that effective treatment targets both the internal (i.e., emotions, traumatic memories) and external (i.e., peer group, drug paraphernalia, certain environments) stimuli that could trigger relapse.

More recently, several EMDR approaches have been developed to treat clients with addictive disorders. All of these have a common approach in that they use desensitization to reduce client's reactivity to situations that might trigger use. However, the targets utilized to accomplish reduced reactivity diverge greatly. In addition, these protocols vary with respect to the requirement (or lack thereof) of client abstinence from substance use.

Omaha (1998) outlined the "Chemotion and EMDR" protocol for treating chemical dependency. Omaha's model seeks to address deficits in identity based on an object relations conceptualization of addiction (Omaha, 1998). After processing resistances to recovery, the protocol utilizes the standard EMDR trauma protocol to target traumatic incidents that are believed to underlie the addiction. After targeting all of the drugs of abuse for a specific client and the associated thoughts/feelings around the trauma, the protocol recommends that the client transition to a counseling group in which additional verbal processing of childhood traumas brings these issues to resolution (Omaha, 1998).

The Desensitization of Triggers and Urge Reprocessing (DeTUR) protocol (Popky, 2005) seeks to reinforce positive coping by focusing clients on both treatment goals and relapse triggers. In the first part of the DeTUR protocol, the client focuses on what is called the Positive Treatment Goal. Each client develops an image of what life would look like when changes are made in alcohol and/or drug consumption, whether the change is abstinence or harm reduction. After enhancing this goal through the use of visual imagery reinforced by bilateral stimulation, the clinician works with the client to develop both internal and external resources to support change. The clinician and client then work to desensitize each trigger for the client's use of substances. Clients are taught to use bilateral stimulation on their own if urges recur.

Vogelmann-Sine, Sine, Smyth, and Popky (1998) published a treatment manual for utilizing EMDR as part of a comprehensive treatment plan for chemical dependency. Extensive assessment of a client's background and current functioning is recommended, including whether a client evidences "affect tolerance, affect containment, and mindfulness" (p. 8). These authors incorporate both the DeTUR protocol (Popky, 2005) and the EMDR Standard Protocol to address triggers of use, that is, thoughts, feelings, and situations, together with traumatic memories that may lead to use. The client's success with managing triggers since the last session is enhanced with eye movements at the beginning of each session.

Zweben and Yeary (2006) outlined how EMDR can be integrated into addictions treatment at different phases. After describing EMDR and addiction treatment, the authors described how the "Safe Place exercise" (Shapiro, 2001) and resource development (Korn \& Leeds, 2002) can be implemented when a client is in early recovery and may be too unstable to do trauma processing (pre-EMDR). The trauma protocol can then be implemented to target earlier traumas that underlie the addiction. These authors noted that abstinence is not necessary to do the trauma work as "many clients may never get clean and sober unless some of the emotional charge is taken out of their traumatic past" (p. 121). Case examples were given to illustrate treatment recommendations.

Hase, Schallmayer, and Sack (2008) target the "addiction memory" in treatment. These authors purport that the memory of addiction, an actual time of use, or a relapse or a craving, may be targeted 
and reprocessed. The protocol used is similar to the Standard Protocol, with the memory of the urge targeted and the rating scale for Level of Urge (LOU) utilized instead of using the Subjective Units of Disturbance Scale (SUDS). Targeting and processing the urge/relapse should also lead to channels back to the original reason the client used substances (Hase, 2006). Ideally, clients will experience a reduction in cravings and a reduced tendency to relapse (Hase, Schallmayer, \& Sack, 2008). Hase (2010) further outlines this protocol with detailed descriptions of how to use this in treatment and has renamed it the CravEx Protocol. It can be a helpful adjunct to EMDR treatment by assisting the client with achieving sobriety in preparation for the Standard Protocol.

\section{Research on EMDR and Addiction}

Research on the clinical efficacy of EMDR with addiction is limited. Hase, Schallmayer, and Sack (2008) published the only randomized control trial found in the literature. EMDR was implemented with or without "treatment as usual." Treatment as usual incorporated detoxification, aspects of motivational interviewing, assessment of social support/adjustment, group treatment, relaxation training, and art therapy. Treatment was for 14-21 days at which time clients were referred to a rehabilitation program and 12-step meetings in their community.

Patients in the experimental group received treatment as usual plus two 1-hr sessions of EMDR that targeted the addiction memory, viewed as the core trigger of relapse. Their findings suggest that EMDR enhanced addiction treatment by decreasing cravings as well as experiences of relapse at 1- and 6-month intervals, respectively, when groups were compared.

Cox and Howard (2007) reported a case study on the use of EMDR with sex addiction. The authors outlined how EMDR can be utilized to treat the early traumas that underlie sex addiction as well as the current situations that trigger relapse. A case study illustrated how EMDR, sex addiction education, 12-step meeting attendance, and work with a sponsor can be combined to treat this disorder. After the client processed past sexual abuse, he was guided in targeting current situations that triggered shame and urges to act out sexually. EMDR was integrated with other therapeutic techniques (letter writing, empty-chair technique, relapse prevention skills) to support ongoing recovery.

Marich (2009) discussed a case exploring the use of EMDR with a cross-addicted female with a history of sexual assault, who met criteria for alcohol dependence, cannabis dependence, sedative dependence, and PTSD. The client presented for treatment after her third Operating a Vehicle Intoxicated (OVI) conviction, having limited success in achieving sobriety (the longest period was 4 months), and using other courses of treatment (both inpatient and outpatient) over a 12-year period of time. After preparing the client for EMDR treatment with a "Safe Place," the author utilized the EMDR Standard Protocol to target shame-based experiences that impeded her recovery. Future template work was then used to reinforce newly developed positive cognitions. Follow-up interviews indicated that the client had successfully maintained sobriety at 18 months.

\section{Case Study}

\section{Introduction to the Case}

This case study illustrates the importance of addressing substance use and abuse, in addition to the clinical dilemma faced by many clinicians as to when or if to use the trauma protocol with an active substance abuser. The authors discuss the factors that were considered in making the clinical formulation and the treatment plan for use of EMDR in this case. The client presented initially with active alcohol dependence as well as PTSD related to the trauma of her husband's suicide. Unessential details were changed to disguise the client's identity and preserve her anonymity.

Presenting Problem. Margo, a widowed, white, 45-year-old woman with three daughters, was referred to this therapist by another therapist in the community. That therapist had been doing couples treatment with Margo and her boyfriend when he discovered Margo's alcoholism and referred her for substance abuse treatment because he felt that this issue was of primary importance. Margo presented to her new therapist with concerns about her increasing use of alcohol. In the past, she had been to several therapists for substance abuse counseling without success. She also had been to Intensive Outpatient (IOP) treatment. She relapsed following each course of therapy. She presented as very frustrated as well as extremely motivated to change these patterns of use. She was a well-educated, professional woman of high intelligence, who was employed full-time. She described herself as isolated and depressed but was not on medications. She experienced anxiety as well as other symptoms of PTSD, including a startle response. She reported that she "did not know who she was." 
Social History. Margo was the oldest of three children who grew up on the West coast of the United States. Father was described as an alcoholic who stopped drinking in college: he was a "dry drunk," that is, an untreated alcoholic who is abstinent from alcohol but who continues to exhibit negative behaviors associated with drinking. Margo reported that he was controlling, negative, and very intelligent. Mother was "nice" and was described as subservient to dad. Margo had a sister who was 4 years older who was reportedly an active alcoholic. Her brother, 2 years younger, gave up alcohol the year before Margo came to this therapist, but continued to smoke marijuana regularly. There was minimal communication in her family of origin and no modeling for appropriate ways to deal with feelings. The family practiced a conservative religion. Growing up in this conservative religious environment was a "horror" for her; she was taught that suffering is good. In high school, Margo spent 6 months living in a religious group home for being "incorrigible." She reported that she had nothing in common with the drug addicts and prostitutes she met there.

After high school, Margo went directly to college where she met her husband. She was married immediately after graduation. She had three daughters, ages 17, 15, and 12. Six years before she came to EMDR treatment her husband committed suicide. He had been depressed for some time but the suicide was completely unexpected. Margo came home and found him in the garage, dead from carbon monoxide poisoning. This was seriously traumatizing to her and she felt grief-stricken, as well as unable to cope with parenting her daughters. Because she had no healthy coping skills, she immediately increased her consumption of alcohol. What she described as heavy social drinking quickly became more regular, heavier, and more abusive. She developed a psychological dependency on alcohol and became unable to stop despite several episodes of therapy and IOP treatment for alcoholism. Her drinking continued to increase, and she wanted to die as she saw no way out of the despair and depression related to her husband's suicide.

\section{Course of Treatment}

When Margo first began treatment with this therapist, it was decided to focus on the alcohol dependence because this was her presenting issue. At the time that she began treatment she had been in therapy with several other providers and had been in IOP treatment. None of these treatment episodes had been successful for her. She was drinking significant amounts of alcohol on an almost daily basis, and presented with symptoms consistent with the diagnosis of PTSD and Alcohol Dependence (APA, 1994).

As a provider for a client who has previously been in many different kinds of treatment and been unsuccessful, it is a challenge to find some new approach to the issue. This client was particularly motivated to change and extremely frustrated by her lack of ability to do so. The therapist in this case situation decided to do something different than what had been tried before, thus the decision to proceed with pre-EMDR and EMDR interventions in addition to a more traditional substance abuse treatment approach. This client was not physically dependent on alcohol, so it was safe to proceed on an outpatient basis.

The treatment, which will be discussed in more detail, included Safe Place, the DeTUR Protocol's Positive Treatment Goal, Resource Development, the Standard Protocol, and Hase's Memory of Addiction Protocol in addition to non-EMDR interventions and self-help groups.

\section{Treatment Focus on Stabilization/Preparation and on Alcohol Dependence}

The first goal of the treatment was client stabilization and preparation for the EMDR work to follow. It was clear from the start that the unresolved trauma and self-blame involving her husband's suicide was contributing to Margo's need to drink. Therefore, it was decided to work with pre-EMDR techniques and traditional substance abuse treatment techniques.

As Shapiro (2001) recommends, treatment started with the Safe Place exercise. This was used to "test" Margo's ability to use EMDR techniques and her responsiveness to them. She was at the point where she was willing to do almost anything. It was somewhat difficult for Margo to find a place where she felt safe and secure, but she came up with an image of a nearby beach. Her cue word was "wave." Once she established this image she worked well with it and felt more relaxed than in the past; she became able to use this to deal with her anxiety in sessions and at home.

This was followed by implementing part of the DeTUR protocol: the positive treatment goal. This protocol is one way to help clients focus on their treatment goals. Installing the goals by using bilateral stimulation reinforces them and provides the client with an image of a new and different way of living. Margo was very clear about wanting to stop drinking. She visualized being abstinent, going through 
her daily life without using alcohol. The therapist reinforced this visualization with short sets of bilateral stimulation, using the audiotac and tappers.

The DeTUR protocol also includes helping the client develop both internal and external resources. Resource Development (Korn \& Leeds, 2002) was utilized to shore up her ability to maintain sobriety. This protocol helps a client develop the ego strength needed to make the changes so important in learning how to live a sober lifestyle. The qualities that Margo chose to reinforce were "strength" and "willpower." She was able to find times earlier in her life when she experienced these qualities, and the audiotac was used for reinforcement. She again visualized an alcohol-free life.

In addition, recovery techniques not related to EMDR or pre-EMDR were utilized. Margo was encouraged to attend AA, to obtain a sponsor, to practice techniques for appropriate affect management, and to identify triggers for her use, as well as techniques to learn how to manage her cravings.

Margo continued to relapse despite efforts to quit using alcohol. After 2 months, she had become extremely upset and frustrated about her inability to stop drinking. Another client may not have been this frustrated and a longer period of time might be needed to get to this point, but Margo was not new to therapy. She was particularly upset because she was so motivated, yet still could not change. After much discussion in treatment, she and her therapist determined that the suicide trauma was interfering with her ability to stay sober. She used alcohol as a way to cope with the feelings resulting from this trauma, and her alcohol consumption increased considerably after the suicide. Her ability to tolerate affect had improved somewhat, as had her internal and external resources (Leeds, 2001), but she had not mastered these skills. Her therapist determined that she would not maintain sobriety without working through her husband's suicide. An important factor in this decision was that Margo had a strong therapeutic alliance with her therapist and was able to use that as a resource. Her sobriety was shaky but she was strongly motivated to change.

\section{Treatment Focus on Traumatic Event}

At the outset of the sixth therapy session, Margo and her therapist decided to begin to work with the trauma protocol. Later, Margo reported that "we jumped into" the trauma work, and that she was glad about this. It took three 1-hr sessions to work through this event. It was clear that the organizing issue was Margo's sense of responsibility for her husband's suicide.

The target was her husband's suicide.

Image: seeing her husband's body in the car in the garage.

Negative cognition: It is my fault.

Positive cognition: It is not my fault.

Validity of Cognition (VoC): 3

Emotions: fear, panic, a need to escape

SUDS: 10

Body: head to chest

In the initial EMDR processing, Margo began by chronologically reviewing the events of the day of the suicide. She expressed considerable emotion. She also processed events that had occurred earlier in the week, including her husband's birthday and some regrets she had about not asking him about how he was doing. She began to see that she had been feeling trapped in the relationship and remembered that she had considered divorce. Her husband's depressive episodes became more obvious to her. With the help of a therapeutic interweave, she was able to see that she did not cause his death. By the close of the session, the SUDS was a 7 out of 10 .

Upon reevaluation at the start of the second session, the SUDS had dropped to a 5. She reported that although she felt sad, there was not the same "punch" to it. In this session, she continued to process events surrounding the suicide and dealing with the reactions of her children and family. Processing proceeded smoothly. The SUDS was 3 at the end of session two.

At the beginning of third session using the Standard Protocol, Margo reported that the week had been a very emotional one. During the week, she felt lonely and wounded, but also pleased about how hard she had worked doing the EMDR processing. She was able to stay sober between sessions. This was difficult but she attended AA, which she had previously resisted, walked a lot, called people, and let herself feel her feelings, which she described as a strange and new experience. The SUDS at the beginning of this session was a 2 . During the processing she described seeing herself coming out of a dark place, as well as her fears about getting close to people. She began to see herself taking down walls. She came up with a dilemma: drinking versus taking the risk of changing and being healthy. The therapist utilized Robin Shapiro's Two-Handed Interweave (Shapiro, 2006). This cognitive interweave can be particularly helpful when a client is ambivalent, torn between two sides of an issue, or feeling stuck. The 
client visualized the drinking in one hand and a different, riskier life in the other. Bilateral stimulation was utilized. The client began to laugh. She saw it as "no contest." Drinking equals death, while not drinking means growing up and being accountable. The SUDS at the end of this session was 0 and the positive cognition was installed. (Note that when reevaluated in ongoing treatment, the SUDS has remained 0.)

\section{Treatment Focus on Early Sobriety and Relapse Prevention}

During the next 6 months, Margo attended treatment with this therapist on a weekly basis. She worked on ongoing sobriety, family and relationship issues, and developed a peer network and social life that she had not had before. Margo seriously began to invest in her sobriety. She attended AA regularly, used a sponsor appropriately, and began to work on the 12 steps. Margo maintained her sobriety for 6 months, until she went on vacation and visited her family. She was severely triggered by the interactions with her family. Upon her return home she went to the grocery store, purchased mouthwash, and consumed it.

\section{Treatment Focus on Addiction Memory}

At the first treatment session following Margo's relapse, the therapist utilized the protocol developed by Hase (2006) to process the memory of addiction. In this case, there was a specific relapse to process. The decision was made to use this protocol because it was specifically developed to use when a client has had a relapse or a craving or urge to relapse. The processing of the Addiction Memory leads the client back to the cause of the addiction, and can be very helpful in terms of the ongoing therapy work. It was a timely intervention, given her recent relapse.

Incident: mouthwash relapse

Image: being in the grocery store ready to pick up the mouthwash

LOU: 10

Negative cognition: I am stupid.

Positive cognition: I am making healthy choices.

VoC: 4

Emotion: shame

Body: core

As processing began, Margo was flooded with feelings. Her abandonment issues surfaced. She felt extremely frightened about opening herself up and risking abandonment; she drank to manage these feelings. She attracted needy people, focused on them, then could not care for herself appropriately. She grew up feeling negated by others and continued to negate herself. After 11 sets of bilateral stimulation, the LOU was 0 , and the positive cognition was installed.

\section{Treatment Focus on Ongoing Relapse Prevention}

That was the last time that Margo relapsed. In subsequent sessions, she did, however, report that she felt cravings and urges on occasion in her life. The Hase Protocol (2006) was utilized four times to deal with these urges. The following is an example of one session after she was triggered by a conversation with her boyfriend:

Image: sitting outside after getting off the phone with her boyfriend

LOU: 8

Negative cognition: I am not lovable.

Positive cognition: I can love myself enough.

VoC: 2

Emotions: disgust

Body: all over

Margo processed this urge to use alcohol for 12 sets of bilateral stimulation. Again, old losses and disappointments emerged, as well as feelings of low selfworth. She saw that she habitually drank to manage these feelings. She also became aware of her ability to make choices and choose healthy responses. At the end of the session, the LOU was a 0 and the VoC was installed.

\section{Follow-Up}

The client utilized the EMDR treatment very successfully and was satisfied with the outcome. The client has continued in therapy until this writing. The focus of treatment has shifted to a focus on past family issues and current relationship problems

Margo wrote a statement, which was read at a local EMDR International Association meeting where this material was presented. She has given the authors permission to include an excerpt here.

After a few (EMDR trauma protocol) sessions I noticed a huge difference. It was like a door opened in the dark room I had locked myself into. For so long I had been unable to get out, afraid of the feelings that would inevitably find me and overwhelm me, driving me back. Since I first started working with (my therapist) my life has changed completely. It has been a lot of 
hard work.... but I no longer wish to die and I

have.... continuous sobriety.

Margo remained sober, and urges to drink alcohol diminished significantly. At this writing she has been abstinent for over 2 years. In addition, she reports little distress related to her husband's death. She continues to grieve normally at holidays, birthdays, and other significant events, but she no longer blames herself nor takes responsibility for his suicide.

\section{Discussion}

\section{Therapy With Addicted Clients}

Addiction is a particularly complex issue for clinicians, due to the multitude of factors involved for each client, including physical, psychological, and social concerns. Dealing with addiction and PTSD (or any other comorbid diagnosis) makes the clinical picture even more complex. This is a field that is changing and growing; there is no one acceptable way to treat this population. It is important to deal with each client individually to maximize results.

\section{Discussion of the Case}

Margo presented to her therapist with concerns about her increasing alcohol consumption. She labeled herself as an alcoholic. She also presented with symptoms of PTSD as a result of her husband's suicide. Although she had been a heavy drinker before his death, she used alcohol to medicate some of these symptoms. It was clear that both these issues would need to be addressed in the therapy. In addition, Margo had several previous attempts at therapy, including IOP Program, which were not successful and which she did not find helpful.

\section{The Decision to Use EMDR Protocols}

As with any therapy, an EMDR clinician must make decisions about how to proceed and what will be the most effective course of treatment. The decision to use EMDR with this client was made, in part, in an attempt to do something different than what had been included in Margo's previous therapies. In terms of EMDR, there are many choices to be made about how to proceed and which protocol to use. Flexibility as a clinician may be the best thing for a client. In this case, the therapist decided to use different parts of a variety of protocols for this reason.

Stabilization/Preparation. Margo benefited from the preliminary work with the Safe Place exercise. She was able to utilize it to calm herself and as a resource to gain peace in her life. The DeTUR Positive Treatment Goal helped her to focus on her goals as well as to actively envision what a different lifestyle might look like. The Resource Development Protocol further shored up her ego strengths and helped prepare her for the trauma work to follow.

Use of the Standard Protocol for Trauma. As the treatment progressed and Margo continued to be unable to stay sober, it became clear to her therapist and to Margo herself that she was haunted by her husband's suicide. Although many substance abuse therapists believe a client should be substancefree before engaging in trauma work (Hase, 2010; Zweben, Clark, \& Smith, 1994), it was determined that this client could not become abstinent without doing the trauma work. This position was supported by Shapiro when she said:

EMDR treatment requires that clinicians recognize that unresolved trauma frequently plays an important role in maintaining substance abuse or dependence... EMDR is used to process these earlier memories, providing the client with a more adaptive perspective of past events and consequently a more functional attitude about the present and future. (Shapiro, Vogelmann-Sine, \& Sine, 1994, p. 383)

Many factors were considered when making this decision:

1. The client's addiction history and patterns of use. Although Margo was a heavy drinker, upon assessment it was determined that she was not physically dependent and would not need a detoxification program. In addition, although she drank before her husband's suicide, the suicide trauma exacerbated her use and it increased markedly after that event.

2. The client's previous attempts to quit using substances. Margo had tried to quit many times and was unsuccessful. She was frustrated by this and willing to try something new and different.

3. The client's motivation. Margo was extremely motivated, despite her frustration.

4. The client's trauma history. Margo had a somewhat neglectful childhood but did not present it as traumatic. Her husband's suicide appeared to be the major trauma in her life. If her trauma history had been more complex, a slower approach, with more emphasis on resource development, may have been necessary.

5. The client's current functioning and stress level. Margo was highly functioning, despite her alcohol 
use. She worked full-time at a professional-level job and maintained some connections with others. Her major stress was the suicide and her use of alcohol.

6. The client's internal/external resources and affect tolerance. This is the one area where the therapist had some concerns about Margo. She was beginning to be able to identify and tolerate her feelings. Her outside supports and relationships were minimal, although she had begun to attend AA. Internally, Margo was just beginning to develop some sense of ego strength, but this was minimal. Leeds (2001) stresses the importance of the client having internal and external resources as well as affect tolerance before proceeding with the Standard Protocol. However, in this case the clinician was a seasoned therapist who felt it would be a detriment to the client not to proceed.

7. The client's use of the therapeutic relationship. This was the key factor in determining to proceed with the Standard Protocol. Margo had a strong therapeutic alliance with her therapist. The therapist was able to promote a sense of safety in the sessions, and the client trusted that the therapist would be her ally in the process.

Use of the Hase Memory of Addiction Protocol. It should be noted that when the therapist began to work with this client, the therapist was not familiar with the work of Michael Hase and processing the addiction memory. This could have been a viable treatment option prior to processing the trauma. Instead, this was used after the trauma work to decrease urges and process the relapse.

It was a logical decision to use the Hase protocol at the time it was used because there had been a recent relapse. It appeared to be more efficient than processing each trigger, as described in the DeTUR protocol. It appears that there may have been some generalizing effect, because after this protocol was used three times the client stopped reporting urges to use alcohol.

\section{Discussion of the Protocols}

Margo responded well to all of the protocols that were utilized. As a result, she was able to markedly change her behavior and become abstinent. Another client may have had more struggles or may have benefited from using these protocols in a different sequence. There is room for much further study about this. The therapist involved in working with a client with comorbid disorders and the use of EMDR must carefully assess each client, each client's needs, and history in order to make the best clinical decision about how to proceed. In addition, the therapist must have the flexibility to change the course of treatment if that becomes necessary. For example, if the client has difficulty processing the trauma work using the Standard Protocol, some additional resource work might be helpful. The Hase protocol may need to be used more frequently with certain clients.

\section{Treatment of Substance Abuse}

The field of addiction treatment continues to mature in terms of what interventions will or will not work for what clients under what conditions. Until recently, "success" in alcohol and other drug abuse treatment has been defined as abstinence from alcohol and mindaltering drugs (Marlatt \& Gorden, 1998). Recent literature recognizes that harm reduction, that is, helping clients to use substances in a way that reduces risk to the client and those around him or her, has gained more acceptance. EMDR holds great promise as a treatment for alcohol and other drug abusers.

\section{Recommendations for Future Research}

Research on both EMDR and addiction treatment will continue to develop. Both qualitative and quantitative designs will be useful to explore ways to assist clients with substance abuse issues. A variety of treatment designs could be utilized.

Quantitative studies could explore how EMDR may enhance addiction treatment in a variety of levels of care (Brown, 2003; Hase, Schallmayer, \& Sack, 2008). Future studies could examine the implementation of various protocols in inpatient, day treatment, intensive outpatient, and outpatient treatment, incorporating when and if to utilize which protocols in these settings. Research might also explore clinical decision-making about when to utilize the DeTUR versus the CraveEx protocol with various clients, as well as combinations of resource development and affect tolerance interventions.

One option might examine looking at two groups of clients with alcohol dependence and PTSD. One group's treatment includes the Standard Protocol and the other's does not. What difference does this make in terms of the client becoming sober and maintaining ongoing abstinence?

Another option would study the use of different types of bilateral stimulation. Eye movements, tones, and tapping could be compared. Which type of bilateral stimulation is most effective with addicted clients? 
Qualitative research could be conducted to investigate and document the unique aspects of using EMDR to treat individuals with substance use disorders. Some questions that might be considered include:

1. How does client motivation for EMDR and readiness to change substance use affect treatment decisions?

2. How do different types of trauma histories (single event versus chronic) affect substance abuse treatment and recovery?

3. In what cases should the clinician use the Standard Protocol first versus using the addiction-oriented protocols first? How does the clinician make the decision to use what part of what protocol with what client and when? This deserves much further study.

4. When should the clinician insist on abstinence in the client prior to trauma work versus working with a harm reduction perspective? Can clients benefit from EMDR at all if they are still using?

\section{Summary}

In this article, the authors have discussed how EMDR is being used with comorbid alcohol dependence and PTSD. The presented case exemplifies some of the clinical issues explored to determine when to use the EMDR addiction protocols and when to use the Standard Protocol with an active substance abuser. The client responded very well to EMDR and has achieved and maintained sobriety. It is, however, a complicated clinical decision as to when and if to use these protocols, and the issue deserves more study. It is an exciting time in the fields of addiction and PTSD treatment. There will prove to be many opportunities to explore possibilities for treatment in both these areas in the 21st century.

\section{References}

Allen, J. P., \& Litten, R. Z. (1999). Treatment of drug and alcohol abuse. An overview of major strategies and effectiveness. In B. S. McCrady \& E. E. Epstein (Eds.), Addictions: A comprehensive textbook (pp. 385-395). New York: Oxford University Press.

American Psychiatric Association. (1994). Diagnostic and statistical manual of mental disorders (4th ed.). Washington, DC: Author.

American Psychiatric Association. (2004). Practice guidelines for the treatment patients with acute stress disorder and posttraumatic stress disorder. Arlington, VA: Author.

Brown, S. (2003). The missing piece: The case of EMDR-based treatment for post traumatic stress disorder and co-occurring substance abuse disorder. Retrieved June 27, 2009, from http://www.lifeforceservices.com/article_detail. php?recordid $=5$

Cox, R. P., \& Howard, M. D. (2007). Utilization of EMDR in the treatment of sexual addiction: A case study. Sexual Addiction and Compulsivity, 14, 1-20.

Foa, E. B., Keane, T. M., Friedman, M. J., \& Cohen, J. A. (2009). Effective treatments for PTSD, second edition: Practice guidelines from the International Society for Traumatic Stress Studies. New York: Guilford.

Ford, J., Hawke, J., Alessi, S., Ledgerwood, D., \& Petry, N. (2007). Psychological trauma and PTSD symptoms as predictors of substance dependence treatment outcomes. Behavior Research and Therapy, 45, 2417-2431.

Gracer, R. I. (2007). A new prescription for addiction (Gracer Behavioral Health Services). San Ramon, CA: Author.

Hase, M. (2006, September). Reprocessing of the addiction memory. Paper presented at the Annual EMDR International Conference, Philadelphia, PA.

Hase, M. (2010). CraveEx: An EMDR approach to treat substance abuse and addiction. In M. Luber (Ed.), EMDR scripted protocols (pp. 467-488). New York: W. W. Norton.

Hase, M., Schallmayer, S., \& Sack, M. (2008). EMDR reprocessing of the addiction memory: Pretreatment, posttreatment and 1-month follow up. Journal of EMDR Practice and Research, 2, 170-179.

Ironson, G., Freund, B., Strauss, J. L., \& Williams, J. (2002). Comparison of two treatments for traumatic stress: A community-based study of EMDR and prolonged exposure. Journal of Clinical Psychology, 58, 113-128.

Jacobsen, L., Southwick, S., \& Kosten, T. (2001). Substance use disorders in patients with post-traumatic stress disorder: A review of the literature. American Journal of Psychiatry, 158, 1184-1190.

Korn, D. L., \& Leeds, A. M. (2002). Preliminary evidence of efficacy for EMDR resource development and installation in the stabilization phase of treatment and complex posttraumatic stress disorder. Journal of Clinical Psychology, 58, 1465-1487.

Lee, C. W., Taylor, G., \& Drummond, P. D. (2006). The active ingredient in EMDR: Is it traditional exposure or dual focus of attention? Clinical Psychology and Psychotherapy, 13, 97-107.

Leeds, A. M. (2001). Strengthening the self: Principles and procedures for creating successful treatment outcomes for adult survivors of neglect and abuse (Cassette Recording and Manual). Santa Rosa, CA: Author.

Marich, J. (2009). EMDR in the addiction continuing care process. Journal of EMDR Practice and Research, 3, 98-106.

Marlatt, G. A., \& Gordon, J. R. (1985). Relapse prevention. New York: Guilford.

Marlatt, G. A., \& Gordon, J. R. (1998). Harm reduction: Pragmatic strategies for managing high risk behaviors. New York: Guilford.

Omaha, J. (1998, July). Chemotion and EMDR: An EMDR treatment protocol based on a psychodynamic model for 
chemical dependency. Paper presented at the 1998 EMDR International Association Conference, Baltimore, MD.

Peirce, J. M., Kindbom, K. A., Waesche, M. C., Yuscavage, A. S., \& Brooner, R. K. (2008). Post-traumatic stress disorder, gender and problem profiles in substance dependent patients. Substance Use and Misuse, 43, 596-611.

Popky, A. J. (2005). DETUR, an urge reduction protocol for addictions and dysfunctional behavior. In R. Shapiro (Ed.), EMDR solutions: Pathways to healing (pp. 167-188). New York: W. W. Norton and Company.

Project Match Research Group. (1997). Project Match secondary a priori hypotheses. Addiction, 92, 1671-1698.

Schumacher, J. A., Coffey, S. F., \& Stasiewicz, P. R. (2006). Symptom severity, alcohol craving, and age of trauma onset in childhood and adolescent trauma survivors with comorbid alcohol dependence and posttraumatic stress disorder. The American Journal of Addictions, 15, 422-425.

Shapiro, F., Vogelmann-Sine, S., \& Sine, L. (1994). Eye movement desensitization and reprocessing: Treating trauma and substance abuse. Journal of Psychoactive Drugs, 26, 379-391.

Shapiro, F. (1989). Eye movement desensitization: A new treatment for post-traumatic stress disorder. Journal of Behavior Therapy and Experimental Psychiatry, 20, 211-217.

Shapiro, F. (2001). Eye movement desensitization and reprocessing (EMDR): Basic principles, protocols, and procedures (2nd ed.). New York: Guilford.
Shapiro, R. (2006). The two-hand interweave. In R. Shapiro (Ed.), EMDR solutions: Pathways to healing (pp. 160-166). New York: W.W. Norton.

Shapiro, F., \& Forrest, M. S. (1997). Eye movement desensitization and reprocessing (EMDR): The breakthrough eye movement therapy for overcoming anxiety, stress, and trauma. New York: Basic.

Solomon, R., \& Shapiro, F. (2008). EMDR and the adaptive information processing model: Potential mechanisms of change. Journal of EMDR Practice and Research, 2, 315-325.

van Wormer, K., \& Davis, D. R. (2008). Addiction treatment: A strengths perspective. Belmont, CA: Thomson Brooks/ Cole.

Vogelmann-Sine, S., Sine, L. F., Smyth, N. J., \& Popky, A. J. (1998). EMDR chemical dependency treatment manual. Honolulu, HI: EMDR Humanitarian Assistance Program.

Zweben, J. E., Clark, H. W., \& Smith, D. E. (1994). Traumatic experiences and substance abuse: Mapping the territory. Journal of Psychoactive Drugs, 26, 327-344.

Zweben, J., \& Yeary, J. (2006). EMDR in the treatment of addiction. Journal of Chemical Dependency Treatment, 8, $115-127$

Correspondence regarding this article should be directed to Nancy J. Abel, Independent Practice, PO Box 2749, South Portland, ME 04116-2749. E-mail: Nanabe150@aol.com 